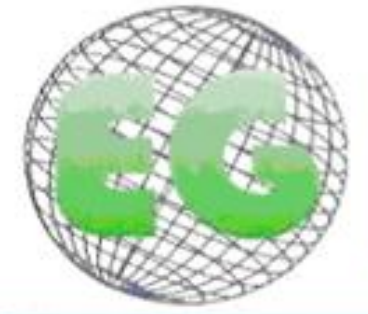

$N^{\circ} 37$

www.um.es/egloball

\title{
CLÍNICA
}

\section{Recogida de urocultivo por sondaje vesical en niños: una observación sistemática}

\author{
Coleta de urocultura por sonda vesical em criança: uma observação sistemática \\ Collect of uroculture by urinary catheter in child: a systematic observation
}

\section{*Guimarães, Marcelle Sampaio de Freitas **Mororó, Deborah Dinorah de Sá **Pinto, Juliana Teixeira Jales Menescal *de Souza, Emeline Noronha Vilar ${ }^{* * *}$ Dantas, Ana Karina da Costa}

\author{
*Residente de Enfermería del Programa Multiprofesional en Salud del Niño de la Universidad Federal \\ de Rio Grande del Norte (UFRN). E-mail: marcelle_sfg@hotmail.com **Máster en Enfermería. \\ Enfermera del Hospital de Pediatría de la UFRN. ***Máster en Pediatría. Residencia Médica Nefrología \\ Pediátrica. Médica Pediatra del Hospital de Pediatria de la UFRN. Natal, Rio Grande do Norte, Brasil.
}

Palabras Clave: Cateterismo Urinario; Infecciones Urinarias; Enfermería Pediátrica.

Palavras-chave: Cateterismo Urinário; Infecções Urinárias; Enfermagem Pediátrica.

Keywords: Urinary Catherization; Urinary Tract Infections; Pediatric Nursing.

\section{RESUMEN}

La infección urinaria se caracteriza por la invasión de microorganismos en el tracto urinario, siendo una de las patologías más frecuentes en todos los grupos de edad, especialmente en niños. El urocultivo se considera el método estándar-oro para el diagnóstico de laboratorio, ofreciendo un alto valor predictivo positivo, si se garantiza una técnica aséptica durante la recolección. El estudio de tipo evaluativo, prospectivo y cuantitativo, tiene por objeto comparar los resultados de los urocultivos por sondaje vesical recogidos por enfermeras del Ambulatorio y Enfermería de un Hospital Universitario Pediátrico teniendo en cuenta el material y la técnica utilizada en el procedimiento.La población de estudio consistió en 12 enfermeros, 4 residentes y los resultados de 300 muestras de cultivos de orina en el período junio-agosto de 2012. Los datos fueron recolectados a través de la observación sistemática y la documentación técnica. Los resultados mostraron una mayor contaminación del procedimiento en la enfermería (13,3\%) que en el Ambulatorio (1,3\%), donde se utilizó kit específico de urocultivo.

\section{RESUMO}

A infecção urinária se caracteriza pela invasão de microrganismos no trato urinário, sendo uma das patologias mais frequentes em todas as faixas etárias, principalmente, em crianças. A urocultura é considerada o método padrão-ouro de diagnóstico laboratorial por oferecer alto valor preditivo positivo, se garantida uma técnica asséptica durante a coleta da urina. O estudo do tipo avaliativo, prospectivo e 
quantitativo objetivou comparar os resultados das uroculturas por sonda vesical coletada por enfermeiros do Ambulatório e Enfermaria de um Hospital Universitário Pediátrico, considerando o material e a técnica utilizada no procedimento. A população estudada foi composta por 12 enfermeiros, 4 residentes de enfermagem e pelos resultados das 300 amostras das uroculturas no período de junho a agosto de 2012. Os dados foram coletados mediante observação sistemática e técnica documental. Os resultados apontaram maior contaminação do procedimento na Enfermaria $(13,3 \%)$ do que no Ambulatório (1,3\%), onde se utilizou kit específico de urocultura.

\section{ABSTRACT}

Urinary tract infection is characterized by invasion of microorganisms in the urinary tract, one of the most frequent pathologies in all age groups, especially in children. The uroculture is considered the gold standard method of diagnosis for offering high positive predictive value, if guaranteed aseptic technique during the urine collected. The assessment study, prospective and quantitative aimed to compare the results of urine cultures for catheter collected by nurses in the clinic and infirmary of a University Hospital Pediatric, considering the material and the technique used in the procedure. The study population consisted of 12 nurses and 4 nursing home residents and the results of 300 samples of uroculture in the period June-August 2012. Data were collected through systematic observation and technical documentation. Results showed higher contamination procedure Infirmary (13.3\%) than in the Clinic (1.3\%), which we used urine specific kit.

\section{INTRODUCCIÓN}

La infección del tracto urinario (ITU) es la invasión y multiplicación de bacterias en el tracto urinario del individuo, lo que puede causar daños en las vías urinarias y en los riñones. Es una de las enfermedades más prevalentes en el mundo, que afecta a todos los grupos de edad, especialmente a las mujeres y grupos de riesgo que incluyen niños, mujeres embarazadas, inmunodeprimidos y personas de edad avanzada que fueron sometidos a cateterismo vesical ${ }^{(1,2)}$.

La Infección urinaria puede ser considerada como un diagnóstico sindrómico ya que comprende varias condiciones clínicas en varios contextos diferentes de presentación ${ }^{(3)}$. Por lo general, la contaminación del tracto urinario se da por vía ascendente, principalmente en las mujeres, debido a la menor extensión de la uretra, y en pacientes sometidos a procedimientos invasivos tales como el sondaje vesical. Pero también puede ser adquirida por vía hematógena a través de la red vascular del riñón, lo que le puede comprometer en cualquier infección sistémica, y raramente por la vía linfática, donde el microorganismo alcanza el riñón por las conexiones linfáticas entre tracto urinario inferior y superior y/o entre el intestino y el riñón ${ }^{(4)}$.

La clasificación de la ITU dependerá de la región afectada. Cuando la participación es en la parte superior se llama pielonefritis aguda y cuando se encuentra en la parte baja del tracto urinario, uretritis o cistitis. La diferencia entre infección urinaria superior e inferior tiene importancia clínica para los niños menores de dos años de edad, pues la presentación clínica de la pielonefritis aguda tiende a no ser específica a esta edad y puede llevar a daño renal ${ }^{(5)}$.

En cuanto a la sintomatología, la ITU puede presentarse de manera diferente en las distintas fases de crecimiento y desarrollo del niño. En el recién nacido se presenta por medio de la ictericia, sepsis, retraso en el crecimiento, regurgitación o vómitos y fiebre. En los bebés y niños en edad preescolar es común ocurra diarrea, retraso del crecimiento, vómitos, fiebre, orina con mal olor, dolor abdominal o en el flanco, incontinencia urinaria, disuria, urgencia para orinar. Ya en los estudiantes y 
adolescentes se suma a los síntomas de niños en edad preescolar el aumento de la frecuencia de micción ${ }^{(6)}$.

El diagnóstico de infección del tracto urinario en los niños puede ser difícil debido los señales de presentación y a que los síntomas que presentan son inespecíficos. Sin embargo, el urocultivo se considera el método estándar-oro de diagnóstico de laboratorio por proporcionar un alto valor predictivo positivo y orientar el enfoque terapéutico correcto. Por lo tanto, es necesario que el profesional responsable de la recogida de la orina asegure la técnica aséptica durante el procedimiento, ya que la fiabilidad de los resultados depende de la recogida apropiada ${ }^{(7,8)}$.

El procedimiento puede ser realizado por sonda vesical, punción suprapúbica, bolsa colectora y jet intermedio. Su elección dependerá en gran medida de la edad del niño y su condición clínica ${ }^{(8)}$.

Para el niño que no puede controlar el esfínter urinario se recomienda el uso de sonda vesical, y en niños de más edad, el colector de orina. La realización de estos procedimientos requiere profesionales cualificados, tanto en la dirección y supervisión del niño y familiar en la recogida de urocultivo por jet medio o bolsa colectora como en la ejecución de la técnica de urocultivo por sonda de vejiga, el cual debe ser realizado por un enfermero o un médico ${ }^{(9)}$.

Vale destacar que corresponde al enfermero la ejecución de cuidados de enfermería técnicamente más complejos y que tengan conocimientos de base científica de conformidad con la Ley $n^{\circ} 7.498 / 86$ que regula la práctica de enfermería ${ }^{(10)}$. El enfermero, a través de su formación, puede contribuir eficazmente a la planificación, ejecución y evaluación de infección urinaria en niños hospitalizados ${ }^{(11)}$.

En el Hospital de Pediatría Profesor Heriberto Ferreira Bezerra (HOSPED) la recogida de urocultivo por sonda vesical se realiza, exclusivamente, por el médico o el enfermero, tanto en la unidad de atención ambulatoria y en la clínica médica y quirúrgica.

EI HOSPED es referencia en la atención especializada a niños y adolescentes en el estado de Rio Grande do Norte. Actúa tanto a nivel ambulatorio como hospital, en una perspectiva multidisciplinar integrando las actividades de enseñanza e investigación. Actualmente, el hospital es el escenario de práctica para los estudiantes de graduación, postgrado y ofrece programas de residencia médica y multiprofesional en pediatría.

La experiencia práctica de residente de enfermaría en el HOSPED, en la unidad de ambulatorios y de hospitalización, llevó a la observación de diferentes técnicas en la recogida de urocultivo por sonda como el material utilizado.

En la unidad de ambulatorio, se observó el uso de un kit de urocultivo compuesto por bandeja riñón, campo fenestrado y gasas. También se utiliza gluconato de clorhexidina, dos guantes estériles, uno para la higiene de la zona genital y otro para introducción de la sonda en la uretra y sonda uretral de cloruro de polivinilo (PVC).

La enfermería no utiliza este kit. Algunos enfermeros organizan el material en la bandeja que contiene un guantes de procedimiento para higiene genital, otro guante estéril para la introducción de la sonda; gasas estériles (para la limpieza de los 
genitales y en sustitución del campo fenestrado), gluconato de clorhexidina y sonda uretral desechable de PVC.

A la luz del contexto presentado, este estudio tuvo como objetivo comparar los resultados de las muestras de urocultivo por sonda vesical recogidas por enfermeros en las unidades de atención de ambulatorio y enfermería del HOSPED, teniendo en cuenta la diferencia en el material y la técnica utilizada.

La relevancia de la investigación radicó en la importancia del procedimiento para el diagnóstico de la infección del tracto urinario, cuya recolección inadecuada puede influir directamente en la fiabilidad de los resultados y en las conductas posteriormente. Además, es un procedimiento invasivo que puede predisponer el paciente a la infección.

\section{MATERIAL Y MÉTODO}

La investigación es de tipo descriptiva, evaluativa y prospectiva, con enfoque cuantitativo y cualitativo. Llevada a cabo en el Hospital Pediátrico Profesor Heriberto Ferreira Bezerra (HOSPED) y en el laboratorio de microbiología de la Maternidad Escola Januário Cicco de la UFRN (MEJC). Ambas son instituciones del Sistema Único de Salud (SUS), pertenecen al Complejo Hospitalario de salud (CHS) de la Universidade Federal de Río Grande do Norte (UFRN) y vinculadas a la Rectoría de la UFRN.

La muestra seleccionada no fue la probabilística, integrado por 12 enfermeros y 4 residentes de enfermería que recogen urocultivos por sondaje vesical en el HOSPED. De estos enfermeros, 3 desarrollan su actividad en ambulatorio y 9 en la enfermería del HOSPED. Sin embargo, se excluyeron dos enfermeras de servicio nocturno, ya que recogen urocultivo por sonda vesical y una residente que estaba con licencia de embarazo.

La investigación también consistió en los resultados de 300 muestras de urocultivos recogidas por sonda vesical, siendo 150 recogidas en el ambulatorio y 150 en la enfermería, en el período de junio a agosto de 2012.

Fueron utilizadas dos técnicas de investigación para recogida de datos: observación sistemática y no participante y la técnica documental. La observación sistemática y no participante fue realizada mediante la aplicación de un cuestionario para trazar el perfil de los investigados y la observación no participante de los enfermeros y residentes de enfermería, a través de la aplicación del instrumento estructurado, anteriormente elaborado por el Comité de Control de Infecciones Hospitalario (CCIH) del HOSPED para el monitoreo de proceso y autorizado para fines de investigación.

La técnica documental fue utilizada para la recogida de los resultados de los urocultivos en el laboratorio de microbiología, utilizando un instrumento desarrollado por los investigadores, que facilitaron informaciones sobre la identificación del paciente, el sexo, la edad, la indicación clínica para el examen y el resultado del examen.

Los datos recogidos fueron analizados utilizando programas estadísticos y mediante el análisis de contenido. Los datos fueron organizados, clasificados y codificados en el software de hojas de cálculo Excel, y analizados de acuerdo a la estadística 
descriptiva, tomando nota la frecuencia absoluta y porcentual, presentada en formas descriptivas, a través de tablas. La discusión y el análisis de contenido se basan en las referencias bibliográficas pertinentes al tema.

Este estudio siguió los principios éticos y legales que rigen la investigación científica con seres humanos. Consistió en la firma del Término de Consentimiento Libre y Esclarecido (TCLE) por los sujetos investigados y responsables de los niños que se sometieron al examen, además se mantuvo el anonimato de las personas que participaron de acuerdo a la Resolución 196/96 del Consejo Nacional de Salud $(\mathrm{CNS})^{12)}$.

El proyecto obtuvo la aprobación del Comité de Ética de la Investigación, bajo parecer fundamentado con registro en el CEP/Documento № no38933 y CAAE no 02772212.3.0000.5292.

\section{RESULTADOS}

La cumplimentación de un instrumento específico, conteniendo preguntas cerradas relacionadas con la vida profesional de los enfermeros, permitió la caracterización de los doces participantes del estudio. Los encuestados tienen edad entre 24 a 55 años, media de 35 años y de un tiempo de formación profesional que va desde 1 hasta 30 años, con una media de 11 años. En cuanto a la relación con la institución, se ha observado que todos los enfermeros son concursados, con jornada de trabajo de 30 horas por semana y sólo dos de ellos tienen otro empleo.

En cuanto al nivel de formación, se observó que una enfermera tiene maestría, 2 son especialistas en enfermería pediátrica, 1 en Unidad de Cuidados Intensivos, 1 Salud Pública, 1 en gestión de hospital, 1 en educación en enfermaría y 4 haciendo aún especialización, siendo 1 en salud del trabajador y las demás en la Salud del Niño. Entre los cursos de actualización realizados por las enfermeras y los residentes, se citaron terapia intravenosa, transfusión y hemoderivados, oncología pediátrica, atención integral a la salud de los niños, y la Gestión Integrada de las Enfermedades Prevalentes de la Infancia (AIEPI).

En las 632 horas de observación sistemática de los 12 enfermeros, señalando que 3 de ellos están trabajando en el sector ambulatorio, fueron acompañados 300 procedimientos de recogida urocultivo por sonda vesical. De éstos, 150 fueron recogidos en el ambulatorio y 150 en la enfermería.

La Tabla I muestra las medidas para la prevención de la infección utilizadas por las enfermeras de la enfermería y ambulatorio de acuerdo con los criterios adoptados por el CCIH del HOSPED para el control de la infección en la instrumentación del tracto urinario referente al procedimiento de cateterismo vesical para recolectar urocultivo por sonda vesical. 
Tabla I - Medidas para prevenir la infección de la recogida de orina por sonda vesical, en el Hospital de Pediatría de la UFRN, en el período junio a agosto de 2012.

\begin{tabular}{|c|c|c|c|c|}
\hline \multirow{3}{*}{$\begin{array}{l}\text { Medidas de Control de } \\
\text { Infección } \\
\text { Higienización de las manos } \\
\text { antes del procedimiento }\end{array}$} & \multicolumn{2}{|c|}{ Enfermería } & \multicolumn{2}{|c|}{ Ambulatorio } \\
\hline & $\mathbf{N}=09$ & $\%$ & $N=03$ & $\%$ \\
\hline & 09 & 100 & 03 & 100 \\
\hline Utiliza Kit de urocultivo & - & - & 03 & 100 \\
\hline $\begin{array}{l}\text { Utiliza materiales sueltos } \\
\text { estériles }\end{array}$ & 09 & 100 & - & - \\
\hline $\begin{array}{l}\text { Abre el material sin } \\
\text { contaminarlo }\end{array}$ & 09 & 100 & 03 & 100 \\
\hline Usa guantes esterilizadas & 07 & 77,8 & 03 & 100 \\
\hline Higiene íntima previa & 09 & 100 & 03 & 100 \\
\hline $\begin{array}{l}\text { Realiza la antisepsia de los } \\
\text { genitales femeninos siguiendo } \\
\text { la secuencia recomendada en } \\
\text { la literatura. }\end{array}$ & - & - & - & - \\
\hline $\begin{array}{l}\text { Realiza la antisepsia de los } \\
\text { genitales masculinos siguiendo } \\
\text { la secuencia recomendada en } \\
\text { la literatura. }\end{array}$ & 05 & 55,5 & 03 & 100 \\
\hline $\begin{array}{l}\text { Utiliza el campo fenestrado en } \\
\text { la región genital. }\end{array}$ & - & & 03 & 100 \\
\hline Identifica material recogido & 09 & 100 & 03 & 100 \\
\hline $\begin{array}{l}\text { Conserva material de urocultivo } \\
\text { en la nevera hasta el envío / } \\
\text { hace envío inmediato }\end{array}$ & 09 & 100 & 03 & 100 \\
\hline $\begin{array}{l}\text { Higieniza las manos después } \\
\text { del procedimiento. }\end{array}$ & 09 & 100 & 03 & 100 \\
\hline
\end{tabular}

Se observa en la Tabla I la falta de uniformidad para la realización de recogida de orina por sonda vesical y la adhesión parcial a las medidas de control de la infección entre los enfermeros en los sectores de ambulatorio y enfermería de la institución. Se constató que los enfermeros siguen parcialmente el protocolo de cateterismo vesical por la $\mathrm{CClH}$ y sólo en el ambulatorio utiliza kit específico para cateterismo vesical, mientras que en la enfermería son utilizados materiales sueltos estériles. Sin embargo, es de destacar que los materiales son abiertos de una manera estéril sin contaminación de los artículos usados. 
En ambos sectores del hospital la realización de la higiene previa y posterior de las manos fue adoptada por todas las enfermeras (100\%). Sin embargo, no hay consenso sobre la adopción de guantes estériles para la higiene de los genitales, porque dos de las enfermeras utilizan guantes no estériles para realizar la higiene de los genitales de los niños $(77,8 \%)$.

Durante el acompañamiento de los procedimientos, se observó que las enfermeras en los dos sectores no siguen la secuencia recomendada en la literatura para la antisepsia de los genitales de los niños, especialmente, las del sexo femenino. Se verificó la predominancia de la secuencia inversa que la literatura recomienda para la antisepsia: meato urinario, los labios menores, labios mayores.

En cuanto a la antisepsia en los genitales masculinos, se observó que el $100 \%$ de las enfermeras del ambulatorio realizan el procedimiento correcto: meato urinario, glande, pene y región púbica, así como el 55,5\% de las enfermeras de la enfermería.

Otro aspecto discutido fue el uso de campo fenestrado. $100 \%$ de las enfermeras hicieron uso del campo fenestrado después de la antisepsia de los genitales y luego se pusieron otro par de guantes estériles. En la enfermería, sin embargo, sólo el $33,3 \%$ de las enfermeras cambiaron los guantes utilizados en la limpieza de los genitales y se pusieron un par de guantes estériles antes de poner el campo estéril, improvisado con gasas estériles.

La identificación de las muestras, de envío inmediato al sector de microbiología o la conservación en la nevera en cuanto espera el envío de la muestra se realizó por el $100 \%$ de las enfermeras, en los diferentes sectores, de acuerdo con el protocolo de la institución y de la literatura científica.

Tabla II - Resultados de los urocultivos recogidos por sonda vesical en el Hospital de Pediatría de la UFRN, en el período junio a agosto de 2012.

\begin{tabular}{lllll}
\hline & \multicolumn{2}{c}{ Enfermería } & \multicolumn{2}{c}{ Ambulatorio } \\
Resultados de los Urocultivos & $\mathbf{N}=\mathbf{1 5 0}$ & $\mathbf{\%}$ & $\mathbf{N}=\mathbf{1 5 0}$ & $\mathbf{\%}$ \\
\hline Proteus Mirabiliis & 01 & 0,7 & 02 & 1,3 \\
Escherichia Coli & 08 & 5,3 & 17 & 11,3 \\
Serratia Marcescens & - & - & 01 & 0,7 \\
Klebsiella Sp & 01 & 0,7 & 03 & 2,0 \\
Pseudomonas Auriginosa & 01 & 0,7 & 01 & 0,7 \\
Morganela Morganii & 01 & 0,7 & - & - \\
Enteropacter sp & 01 & 0,7 & - & - \\
Enterococcus & - & - & 03 & 2,0 \\
Ausencia de Crecimiento Bacteriano & 117 & 78,0 & 121 & 80,7 \\
Contaminación de las muestras & 20 & 13,3 & 02 & 1,3 \\
\hline
\end{tabular}

En la Tabla II, podemos observar las frecuencias absolutas y porcentajes de los resultados de los urocultivos recogidos durante el período de junio a agosto, en los sectores estudiados. Se constata que la contaminación de las muestras fue más común en la enfermería (13,3\%), que utiliza materiales estériles sueltos que en el ambulatorio (1,3\%), que hace uso de Kit de urocultivo especifico para este examen. La contaminaciones de las muestras fueron debido a la presencia de colonias mixtas $(40,7 \%), \quad 0$ agentes aislados tales como Staphylococcus aureus $(22,7 \%)$ y Staphylococcus sp coagulase negativo $(36,6 \%)$, que normalmente colonizan la piel. 
El microorganismo más frecuentes en el tracto urinario de los niños hospitalizados fue la E.Coli $(5,3 \%)$; mientras que en el ambulatorio, fueron la E.Coli $(11,3 \%)$, Klebsiella sp (2,0\%), Enterococcus (2,0\%), Proteus Mirabiliis (1,3\%).

\section{DISCUSIONES}

Algunos aspectos relacionados con el perfil de los investigados se pueden reflejar en la práctica profesional. Uno de ellos es el factor de la edad y el tiempo de formación, donde ambos presentan variaciones que se deben a la presencia de enfermeros que vienen de una formación más antigua y a la participación de los residentes de enfermería en el estudio. Este hecho es un aspecto positivo, ya que permite un intercambio de conocimientos, teniendo en cuenta que el enfermero, con sus vastas experiencias prácticas, ayudó en la formación de los residentes, y estos por ser recién graduados, comparten los conocimientos adquiridos en su reciente formación, generando reflexiones y discusiones acerca de su hacer.

En cuanto al nivel de formación, se nota la creciente preocupación de los enfermeros con la cualificación profesional atribuida posiblemente a las demandas del mercado laboral, a los avances tecnológicos en salud y por estar insertos en un hospital universitario. Aunque se observa una preferencia por la mejora técnica especializada y curativa, también se señaló el interés de los enfermeros en la realización de cursos que reflejan el Sistema Único de Salud (SUS).

La prevalencia de enfermeros para el vínculo exclusivo y estable puede ser un factor positivo en su práctica asistencial, ya que son profesionales que experimentan el pleno empleo y tienen disponibilidad para la formación continua en salud.

En relación a la práctica asistencial de enfermería para recogida de orina por sonda vesical para urocultivo, fue posible constatar la falta de uniformidad en la aplicación de este procedimiento por las enfermeras de la institución, lo que se reflejó directamente en los resultados de la investigación.

El uso de kit especifico para urocultivo por cateterismo vesical fue adoptado solo en la unidad de ambulatorio, pero el resultado del estudio indica la necesidad de un kit estéril que contenga los siguientes materiales: cuba riñón, fórceps, gasas y campo fenestrado, ya que el uso de materiales sueltos estériles permite romper la cadena aséptica $^{(8,13)}$.

La higiene de manos antes y después de los procedimientos es esencial para prevenir las infecciones cruzadas y hospitalarias ${ }^{(14)}$, se observó una adhesión de las enfermeras del estudio a esta práctica durante la recolección de orina para urocultivo.

La literatura recomienda el uso de guantes estériles y la adopción de una técnica aséptica durante la realización de un cateterismo vesical ${ }^{(13)}$. Sin embargo, los resultados de esta investigación indican la necesidad de una cualificación profesional, ya que algunas enfermeras no usaron guantes estériles para higienizar los genitales de los niños, como también demostraron una falta de uniformidad en la técnica de antisepsia.

Es de destacar, sin embargo, que el antiséptico influye directamente en el resultado del examen, por esta razón, durante la realización es necesario observar los siguientes principios asépticos: la fricción y la única dirección (anterior a posterior). La literatura informa que la secuencia apropiada para la antisepsia es: meato urinario, 
labios menores, labios mayores en las mujeres, y meato urinario, glande, pene y región púbica en los hombres. Una vez que se define la orden de ejecución del procedimiento a partir de la zona menos contaminada a la zona de contaminación más alta ${ }^{(13)}$.

Otro aspecto discutido fue el uso de campo fenestrado, cuyo propósito es asegurar una técnica aséptica. Debe ser colocado después de la limpieza de los genitales, con el uso de guantes estériles de modo que su superficie no sea contaminada ${ }^{(9,13)}$.

En este estudio se encontró que el campo estéril sólo está disponible en la unidad de cuidados ambulatorios, donde se utiliza el kit de cultivo de orina. En enfemería se utilizan gasas estériles para reemplazar el campo. Sin embargo, hubo un mal manejo de estas por el 66,7\% de las enfermeras, por no haber cambiado los pares de guantes estériles antes de la manipulación de las gasas. Por lo tanto, se supone que hubo contaminación de las gasas por los guantes utilizados en la antisepsia de los genitales del niño, por lo que pudo haberse producido también la contaminación de la sonda uretral, y de la orina recogida.

Por otra parte, se constató a través de la observación, que las muestras de orina recogidas por sonda vesical se enviaro al laboratorio de microbiología en el tiempo hábil o almacenadas en el refrigerador apropiado, en consonancia con la literatura científica. En este sentido, se percibe la identificación de las muestras recogidas, y su envío lo antes posible al laboratorio de microbiología. El tiempo recomendado entre la recogida y la entrega de la muestra en el laboratorio no debe exceder de una hora; sin embargo, en caso de imposibilidad de envío inmediato, debe mantenerse la muestra en un refrigerador adecuado, a temperatura entre 2 a $5^{\circ} \mathrm{C}$, en el máximo, por 24 horas $^{(15-16)}$.

Los principales patógenos implicados en las infecciones del tracto urinario en niños menores de 12 años son la Escherichi coli, os Proteus mirabilis e vulgaris, a Morganella morganni, a Kleibsiella pneumoniae e o Streptococcus $s p^{(2)}$. En este estudio, el principal patógeno responsable de la infección del tracto urinario fue la Escherichi coli. Sin embargo, los estudios muestran que este índice es significativo, pero no el más frecuente en los casos de infección urinaria ${ }^{(16)}$.

\section{CONCLUSIONES}

El estudio mostró una mayor contaminación en las muestras de urocultivo recogidas en el sector de enfermería en comparación con las recogidas en la unidad de ambulatorio, que se utiliza el kit de urocultivo para realizar el procedimiento.

Se puede concluir, por tanto, que este kit ha influido positivamente en la ejecución de la técnica de recogida de urocultivo por sonda y, consecuentemente, en la calidad de las muestras de orina, constatándose la importancia de la normalización en todas sus unidades de atención de la institución.

Sin embargo, se identificaron puntos críticos en el cotidiano de la práctica del enfermero durante la recogida de orina por sonda que puede haber influido en la fiabilidad de los resultados de los urocultivos y causado la contaminación de las muestras. 
Además, se verificó, a través de la observación directa de los enfermeros durante la ejecución de la técnica de recogida de orina por sonda, que la mayoría de ellos se adhiere a las medidas básicas y fundamentales para la prevención y control de infecciones. Entre estas medidas, se destaca el lavado de las manos, que se realizó antes y después del procedimiento por todos los sujetos de la investigación.

Se resalta, también, la importancia de la integración entre la teoría y la práctica para el ejercicio de la enfermería, donde es necesario la accesibilidad a un protocolo de atención respaldado científicamente, con el fin de estandarizar los procedimientos realizados por el equipo.

Las medidas educativas y de capacitación para todos los enfermeros son vitales para mejorar la técnica, corregir los errores aún presentes en la práctica y, en consecuencia, reducir al mínimo los niveles de contaminación de las muestras recogidas.

El enfermero, como líder del equipo, debe estar atento a las innovaciones inherentes a su saber hacer, reevaluando y elaborando normas y rutinas, y también implementando la educación en servicio para calificar la asistencia y cuidados prestados.

Teniendo en cuenta lo anterior, hemos identificado la necesidad de estandarización de la técnica realizada por los enfermeros de la institución, ya que es un Hospital de la Universidad responsable por la formación de los profesionales de la salud.

Esta investigación se tornar relevante, ya que demuestra evidencia científica para la práctica del enfermero y así podrá contribuir a la normalización de los Protocolos Operativos Estándar (POE), lo que contribuye significativamente a la enseñanza, investigación y asistencia.

\section{REFERENCIAS}

1. Nishiura JL, Heilberg IP. Infecção urinária. São Paulo: Ed. Moreira Jr. 2009.

2. Araujo KL, Queiroz AC. Análise do perfil dos agentes causadores de infecção do trato urinário e dos pacientes portadores, atendidos no Hospital e Maternidade Metropolitano-SP. J Health Sci Inst. [periódico na Internet]. 2012 [citado 20 jan 2013]; $30 \quad$ (1), p.7-12. Disponível em:http://www.unip.br/comunicacao/publicacoes/ics/edicoes/2012/01 janmar/V30 n1 2011 p7-12.pdf.

3. Costa LC, Belém LF, Silva PMF, Pereira HS, Silva Júnior ED, Leite TR, et al. Infecções urinárias em pacientes ambulatoriais: prevalência e perfil de resistência aos antimicrobianos. Campina Grande: RBAC. 2010; vol. 42(3): p.175-180.

4. Silveira AS, Araújo MC, Fonseca FM, Okura MH, Oiveira ACS. Prevalência e Suscetibilidade Bacteriana em Infecções do Trato Urinário de Pacientes Atendidos no Hospital Universitário de Uberaba. RBAC [periódico na Internet]. 2010 [citado 19 jan 2013]; $42 \quad$ (3), p.157-160. $\quad$ Disponível http://www.sbac.org.br/pt/pdfs/rbac/rbac 42 03/rbac 42 v3_001.pdf.

5. Garin EH, Olavarria F, Araya C, Broussain M, Barrera C, Young L. Diagnostic significance of clinical and laboratory findings to localize site of urinary infection. Florida: Pediatr Nephrol. 2007; 22: p.1002-1006.

6. Doyne E, Voet S, Frederic S, Defoor W, Gelfond M, Jackson E., et al. Evidencebased care guideline for medical management of first urinary tract infection in children 
12 years of age or less. Cincinnati $(\mathrm{OH})$ : Cincinnati Children's Hospital Medical Center. Nacional Guideline Claringhouse. 2008, p.1-21.

7. Cunha O, Garrido A, Gonçalves M, Ferreira G, Marques E, Vilarinho A. Utilidade da urocultura de controlo na infecção urinária. Rio de Janeiro: Acta Pediatr. Port. 2010; 21 (2): p.51-3.

8. Carvalhal GF, Rocha LCA, Monti PR. Urocultura e exame comum de urina: considerações sobre sua coleta e interpretação. Revista da AMRIGS. 2006; 50 (1): p.59-62.

9. Mayorga IJ, Sánchez MS, Carrasco LV, Morales JC, Hidalgo LR, Carreño RC, et al. Protocolo de Sondaje Vesical. Biblioteca Lascasas [periódico na Internet]. 2010 [citado 01 nov 2012]; 6(1): 23p. Disponível em: http://www.indexf.com/lascasas/ documentos/lc0509.pdf.

10. Brasil. Decreto-lei o 7.498, de 25 de junho 1986. Institui a Lei do exercício da profissão de enfermagem. Lex: Conselho Federal de Enfermagem: edição federal, Brasília, DF, 25 de junho 1986. Seção 1, $9273-9.275 p$.

11. Queirós MI, Cipriano MAB, Santos MCL, Cardoso MVLML. Infecções urinárias e uso de cateter vesical de demora em unidade pediátrica. Rev Rene. 2011; 12(2):295301.

12. Conselho Nacional de Saúde (BR). Resolução 196 de 10 de outubro de 1996. Diretrizes e normas regulamentadoras de pesquisa envolvendo seres humanos. Bioética. 1996; 4 (2 supl.): 15-25.

13. Souza ACS, Tipple AFV, Brabosa JM, Pereira MS, Barreto RASS. Cateterismo urinário: conhecimento e adesão ao controle de infecção pelos profissionais de enfermagem. [periódico na Internet]: Revista Eletrônica de Enfermagem. 2007 [citado 22 out 2012]; 9 (3): $\quad$ p. 724-735. Disponível em: http://www.fen.ufg.br/revista/v9/n3/pdf/v9n3a12.pdf.

14. Felix CCP, Miyadahira AMK. Avaliação da técnica de lavagem das mãos executada por alunos graduando do curso de graduação em enfermagem. Revista da Escola de Enfermagem USP. 2009; 43(1): p.139-145.

15. Brasil. Ministério da Saúde. Manual de procedimentos básicos em microbiologia clínica para o controle de infecção hospitalar: Módulo I/Programa Nacional de Controle de Infecção Hospitalar - Brasília: ANVISA / 2004.

16. Salvador PTCO, Alves KYA, Dantas RAN, Dantas DV. Infecção do trato urinário relacionada ao cateterismo vesical: revisão integrativa da literatura. Rev Enferm UFPE [periódico na Internet]. 2010 [citado 03 nov 2012]; 4(3): p.954-961. Disponível em: http://www.ufpe.br/revistaenfermagem/. 


\section{ANEXO A}

INSTRUMENTO PARA COLECTA DOCUMENTAL

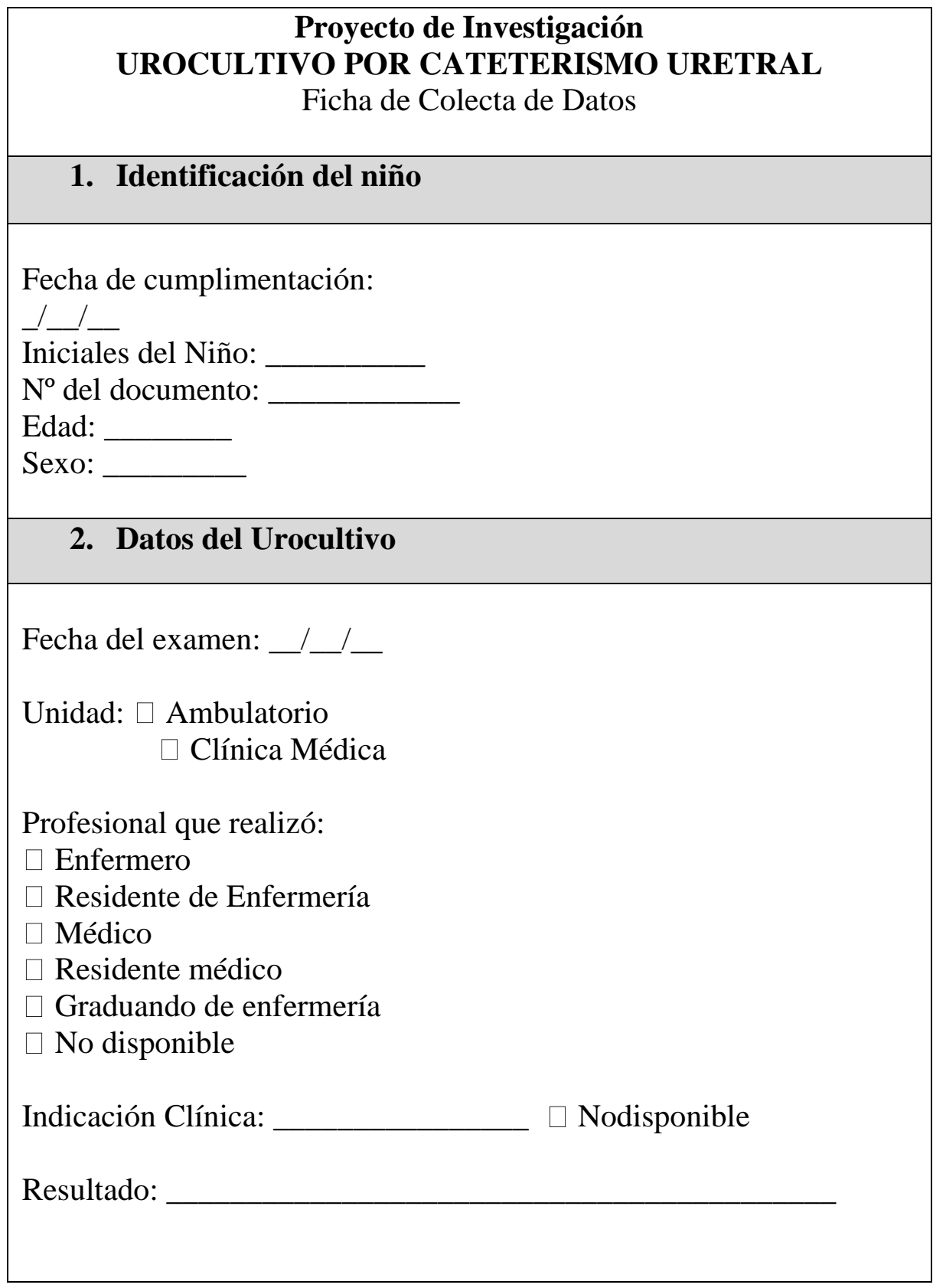




\begin{tabular}{|c|c|c|c|}
\hline \multicolumn{4}{|c|}{$\begin{array}{c}\text { Proyecto de Investigación } \\
\text { UROCULTIVO POR CATETERISMO URETRAL } \\
\text { INSTRUMENTO ESTRUCTURADO PARA MONITORIZAR EL PROCESO DE } \\
\text { COLECTA DE UROCULTIVO POR SONDA VESICAL }\end{array}$} \\
\hline \multicolumn{4}{|l|}{ FECHA: } \\
\hline CRITERIOS ADOPTADOS EN EL PROCESO & SI & NO & OBSERVACIONES \\
\hline $\begin{array}{l}1 . \mathrm{O} \text { ¿Enfermero higieniza las manos antes de iniciar el } \\
\text { procedimiento? }\end{array}$ & & & \\
\hline 2. Explica el procedimiento al niño o acompañante? & & & \\
\hline 3. ¿Acomoda el niño en la posición adecuada? & & & \\
\hline 4. ¿Promueve la privacidad durante el procedimiento? & & & \\
\hline 5. ¿Abre el material a ser utilizado sin contaminarlo? & & & \\
\hline $\begin{array}{l}\text { 6. ¿Se coloca los guantes estériles tras la higiene de las } \\
\text { manos? }\end{array}$ & & & \\
\hline $\begin{array}{l}\text { 7. ¿Se pone los guantes de procedimiento (no estéril) tras la } \\
\text { higiene de las manos? }\end{array}$ & & & \\
\hline $\begin{array}{l}\text { 8. ¿Utiliza Kit Urocultivo (cuba riñón, campo fenestrado, } \\
\text { gasas estériles)? }\end{array}$ & & & \\
\hline $\begin{array}{l}\text { 9. ¿Realiza antisepsia de los genitales con solución } \\
\text { fisiológica y clorexidine antigérmenes, ejecutando } \\
\text { movimiento en sentido único? }\end{array}$ & & & \\
\hline $\begin{array}{l}\text { 10. ¿Retira los guantes usado para antisepsia de los } \\
\text { genitales y usa los guantes estériles? }\end{array}$ & & & \\
\hline 11. ¿Utiliza campo fenestrado? & & & \\
\hline 12. ¿Utiliza gasas estériles como campo? & & & \\
\hline $\begin{array}{l}\text { 13. ¿Realiza introducción de la sonda y procede a colceta de } \\
\text { la muestra de forma estéril? }\end{array}$ & & & \\
\hline $\begin{array}{l}\text { 14. ¿Retira los guantes tras el procedimiento, e en seguida } \\
\text { higieniza las manos? }\end{array}$ & & & \\
\hline 15. ¿Deja al paciente confortable? & & & \\
\hline $\begin{array}{l}\text { 16. ¿Envía el material al laboratorio debidamente } \\
\text { identificado? }\end{array}$ & & & \\
\hline $\begin{array}{l}\text { 17. ¿Almacena en el refrigerador la orina recogida ante la } \\
\text { imposibilidad de envío inmediato al laboratorio? }\end{array}$ & & & \\
\hline
\end{tabular}




\section{ANEXO C \\ UNIVERSIDADE FEDERAL DO RIO GRANDE DO NORTE \\ CENTRO DE CIENCIAS DE LA SALUD \\ RESIDENCIA MULTIPROFESIONAL EN SALUD \\ CUESTIONARIO PARA TRAZAR EL PERFIL DEL INVESTIGADO}

Este instrumento tiene como objetivo definir el perfil del enfermero que realiza la técnica de urocultura por sonda vesical para ayudar el trabajo "Evaluación de la técnica de recogida de uroculivo por sonda vesical en un hospital universitario pediátrico".

Residente: Marcelle Sampaio de Freitas Guimarães

Orientadora: Enf ${ }^{a}$ Ms.Deborah Dinorah de Sá Mororó

Fecha:

Lugarl:

1 - DATOS DE IDENTIFICACIÓN

1.1 Edad:

1.2 Sexo: ( ) masculino ( ) femenino

1.3 Formació: ( ) graduación Tempo de formación:

1.4 Institución de Formación: ( ) pública ( ) privada

1.5 Post-graduación: ( ) especialización

$$
\begin{aligned}
& \text { ( ) máster } \\
& \text { ( ) doctorado }
\end{aligned}
$$

1.6 Institución de Formación: ( ) pública ( ) privada

1.7 Tiempo de Institución: Vínculo de trabajo: ( ) sí ( ) no

1.8 Carga horaria semanal:

1.9 Principales cursos de actualizació que usted realizó, en qué año:

2. Otros vínculos: ( ) sí ( ) no

2.1 En caso afirmativo ( ) Institución privada ( ) Institución pública 
Recibido: 9 de mayo de 2013; Aceptado: 14 de julio de 2013 